\title{
Effectiveness of electroacupuncture for polycystic ovary syndrome: study protocol for a randomized controlled trial
}

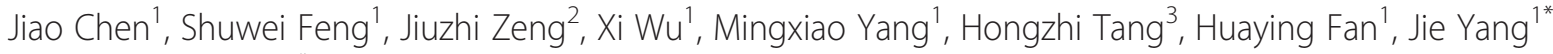 \\ and Fanrong Liang ${ }^{1 *}$
}

\begin{abstract}
Background: Whether electroacupuncture is effective for patients with polycystic ovary syndrome is still inconclusive. Therefore, this study aims to evaluate the add-on effects of electroacupuncture to conventional drugs for the treatment of polycystic ovary syndrome.

Methods/design: This study is a two-center, open-labeled, randomized, controlled trial. A total of 116 eligible patients with polycystic ovary syndrome will be randomly allocated in a 1:1 ratio to the electroacupuncture plus clomiphene citrate group or to the clomiphene citrate group. Participants in the electroacupuncture plus clomiphene citrate group will receive electroacupuncture treatment in addition to clomiphene citrate capsules, whereas participants in the clomiphene citrate group will be prescribed clomiphene citrate capsules only. Electroacupuncture treatment will be performed from the fifth day of menstruation or withdrawal bleeding until the next menstruation, in three sessions per week for three menstrual cycles. The primary outcome is the ovulation rate. The secondary outcomes include the dominant follicle rate, mean number of dominant follicles, endometrial thickness, time point of ovulation, follicular size before ovulation, luteinizing hormone, estradiol level, and pregnancy rate. The measuring points for outcomes will be baseline and the completion of treatment. Any adverse events occurring during the trial process will be recorded. In addition, a quality-monitoring group independent from the research team will be set up to control the quality of the trial.

Discussion: The design and methodological rigor of this trial will allow for the collection of valuable data to evaluate the effectiveness of electroacupuncture for treating polycystic ovary syndrome. Therefore, this trial will contribute reliable evidence for use in clinical decision-making in acupuncture therapy of polycystic ovary syndrome as well as to future research in acupuncture for polycystic ovary syndrome.
\end{abstract}

Trial registration: Chinese Clinical Trial Registry, ChiCTR-IOR-15007358, registered on 26 October 2015

Keywords: Electroacupuncture, Polycystic ovary syndrome, Effectiveness, RCT, Study protocol

\section{Background}

Polycystic ovary syndrome (PCOS), resulting from an imbalance of hormones, is a common disease in women. Its clinical manifestations vary, but include reproductive symptoms (hirsutism [1], infertility [2], pregnancy implications, and neonatal complications [3]) and metabolic complications (obesity [4], impaired glucose tolerance,

\footnotetext{
*Correspondence: jenny_yang_jie@126.com; acuresearch@126.com ${ }^{1}$ Chengdu University of Traditional Chinese Medicine, Chengdu, Sichuan, China

Full list of author information is available at the end of the article
}

metabolic syndrome [5], and type 2 diabetes mellitus [6]). Usually, many psychological comorbidities accompany PCOS, including depression, anxiety, moodiness, panic attacks, body dissatisfaction, eating disorders, and a lower health-related quality of life [7, 8]. As one of the common causes of anovulatory infertility, PCOS accounts for a significant proportion (40\%) of all women who receive infertility treatment [2]. More than 116 million (3.42 \%) women worldwide are affected by PCOS [9]. Using the Rotterdam criteria, the prevalence has been as high as $17.8 \pm 2.8 \%$ [10]. 
Currently, first-line conventional therapy for infertile women with PCOS is the oral administration of antiestrogen agents (for example, clomiphene citrate). Nevertheless, from a clinical point of view, the current management of PCOS with these drugs is not always satisfactory and produces some obvious side effects. After taking clomiphene citrate, metformin, or both, $41 \%, 76.3 \%$, and $31.6 \%$ of women, respectively, still failed to ovulate [11]. The live-birth rate for these patients was only $22.5 \%$ after taking clomiphene citrate, $7.2 \%$ after taking metformin, and $26.8 \%$ after taking both clomiphene citrate and metformin. Among pregnancies, clomiphene citrate caused multiple pregnancies in $6.0 \%$ of the cases. Other side effects include gastrointestinal symptoms, hot flashes, and symptoms associated with ovarian enlargement and ovulation [12].

Whereas first-line conventional therapy is not satisfactory, acupuncture, as a nonpharmacological therapy, is gaining popularity. Findings from previous clinical trials suggest acupuncture may induce ovulation [13]; improve menstrual frequency [14]; and improve depression, anxiety, and health-related quality of life [15] in PCOS patients. Some of the mechanisms underlying acupuncture as a treatment for PCOS have been identified. Acupuncture is able to decrease abnormally high levels of circulating luteinizing hormone (LH), which affect the $\mathrm{LH} /$ follicle-stimulating hormone (FSH) ratio $[14,16]$, and high levels of testosterone [17]. Additionally, the low associated adverse-events rate, a low risk of multiple pregnancies, and the low cost of acupuncture have been noted [18]. However, the methodological quality of these clinical trials varies, resulting in inconclusive statements on the effectiveness of acupuncture for PCOS. In this case, we designed this two-center, open-labeled, randomized controlled trial (RCT) to evaluate the add-on effects of electroacupuncture to conventional drugs on ovulation and some sex hormones in women with PCOS.

\section{Methods/design}

\section{Overview of the study design}

We designed an open-labeled RCT. The interventions will be performed under ideal circumstances. Therefore, this trial will be carried out in academic settings: the First Affiliated Hospital of Chengdu University of Traditional Chinese Medicine and the Third Affiliated Hospital of Chengdu University of Traditional Chinese Medicine. The flow is shown in Fig. 1.

Before inclusion, participants will be screened in the aforementioned research centers. If the participants meet the inclusion criteria and agree to participate, they will be asked to sign the informed consent form and, then, will be randomized into the electroacupuncture plus clomiphene citrate group (EA group) or the clomiphene citrate group (CC group).

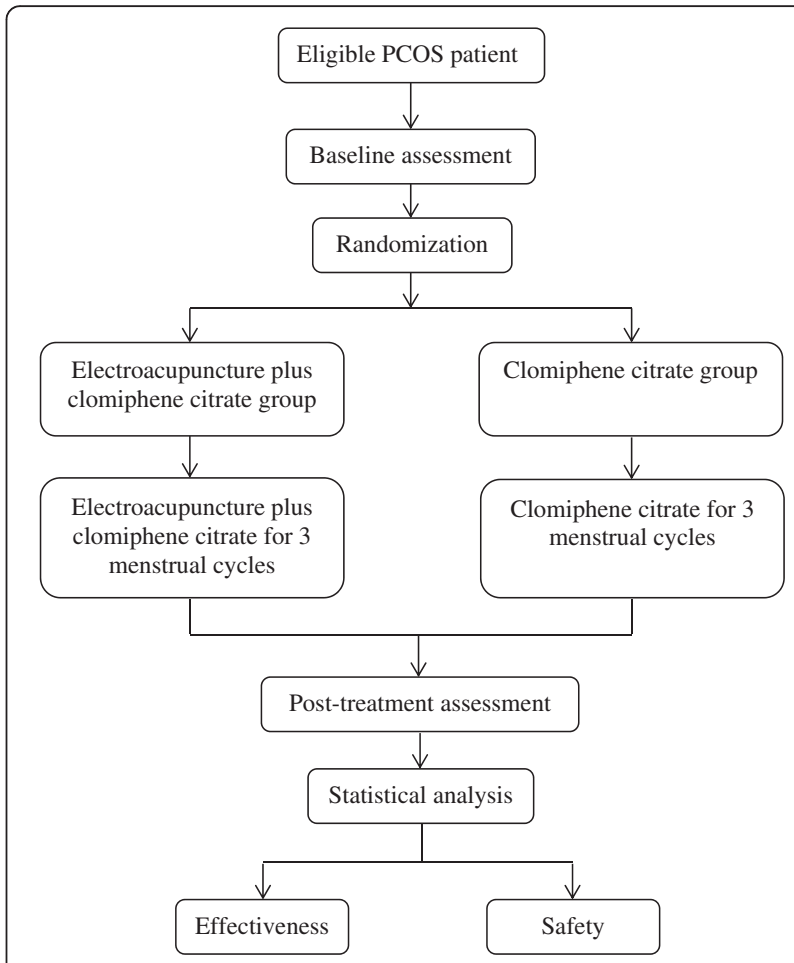

Fig. 1 Flow chart of the trial

After randomization, the participants will go through a total treatment period of three menstrual cycles. Outcome measurements will be assessed by assessors, blinded to the group assignment, at baseline and the end of treatment. Adverse events will be recorded for the assessment of safety. In addition, a quality monitoring board will be set up to control the quality of the protocol implementation.

The study protocol has been reviewed and approved by local institutional review boards and ethics committees. It follows the principles of the CONSORT and STRICTA statements as well as the Declaration of Helsinki (Sixth revision, 2008). Informed consent will be obtained from all participants.

\section{Ethics approval}

All trial procedures place the participant's benefit as the highest priority. The present study protocol has already been ethically approved by the Sichuan Regional Ethics Review Committee on Traditional Chinese Medicine with the ethical approval identifier 2015KL-009.

\section{Participants}

We plan to recruit patients with PCOS diagnosed on the basis of Revised 2003 Consensus on Diagnostic Criteria and Long-Term Health Risks Related to Polycystic Ovary Syndrome established by The Rotterdam ESHRE/ASRMSponsored PCOS Consensus Workshop Group [19]. 
According to these criteria, women with two out of three of the following items can be diagnosed as having PCOS: (1) oligoovulation or anovulation, (2) clinical and/or biochemical signs of hyperandrogenism, and (3) polycystic ovaries and exclusion of other etiologies (congenital adrenal hyperplasia, androgen-secreting tumors, Cushing's syndrome). Further assessment of eligibility is based on the inclusion and exclusion criterion as follows.

\section{Inclusion criteria}

1. Female, age 20 to 40 years

2. Diagnosed as having PCOS

3. Sign the inform consent

\section{Exclusion criteria}

1. Allergic constitution or allergic to various kinds of medicine

2. Hyperandrogenism due to other reasons, including hyperprolactinemia, thyroid disease, congenital adrenal hyperplasia, Cushing's syndrome, etc.

3. Pathological endometrial changes, such as uterine malformation, hysteromyoma, etc., diagnosed by B ultrasound

4. Genital tract malformation, gonadal dysgenesis, fallopian tube block, etc.

5. Severe heart, liver, or renal dysfunction or hematological, respiratory, cardiovascular, psychiatric, or other metabolic diseases

6. Within 6 weeks post-abortion

7. Use of hormonal or other medication including Chinese herbal prescriptions in the past 3 months

8. Participation in other clinical trials at the same time

\section{Randomization}

In this trial, participants will be randomly assigned to either the EA group or the CC group in a 1:1 ratio using a table of random numbers. The random numbers are generated using SAS statistical software package (Version 9.1, SAS Institute Inc.).

\section{Blinding}

This is an open-labeled trial; therefore, neither the patients nor the clinical practitioners will be blinded for the entire process. However, in order to eliminate potential bias, other researchers, including data collectors and statisticians, will be blinded.

\section{Interventions}

Participants in the EA group will receive electroacupuncture treatment in addition to the oral administration of clomiphene citrate, whereas participants in the
CC group will receive clomiphene citrate only. Treatment in both group will last three menstrual cycles. The intervention in this trial involves a rigorous acupuncture schedule. To ensure the compliance of acupuncturists to the schedule, we ask them to take pretrial training and an entrance exam for this trial. All included acupuncturists will have at least 2 years of practical experience in clinical acupuncture.

\section{Clomiphene citrate therapy}

On the fifth day of menstruation, all participants, except for those with amenorrhea, will receive a pharmacological treatment regimen of $50 \mathrm{mg}$ clomiphene citrate, taken once each day for 5 continuous days. Patients with amenorrhea will receive the same pharmacological treatment regimen after withdrawal bleeding has been induced by taking progestin. In cases of no ovulation, an additional $50 \mathrm{mg}$ will be added to the dose in the next menstrual cycle. The maximum dose will not exceed $150 \mathrm{mg}$. The therapy will last for three menstrual cycles.

\section{Electroacupuncture}

In order to increase the reproducibility and internal validity of the trial, electroacupuncture treatment will be applied to PCOS patients. As the stimulation of electroacupuncture can be more quantifiable relative to manual acupuncture, its employment may reduce the risk of bias somewhat. Sanyinjiao (SP 6), Guanyuan (BL 26) Zhongji (CV 3), and Zigong (EX-CA 1) have been chosen for the formula of compulsory acupoints. Additionally, sets of three arbitrary points will be alternately selected according to the phase of the menstrual cycle: Taichong (LR 3) and Taixi (KI 3) will be needled after menstruation; Mingmen (GV 4) and Xuehai (SP 10), during the ovulatory period; and Xuehai (SP 10) and Geshu (BL 17), before the next menstruation.

Each acupoint will be needled using a filiform needle (25-50 $\mathrm{mm}$ in length and $0.25 \mathrm{~mm}$ in diameter) to achieve a Deqi sensation (refers to a sensation of numbness, distension, or electrical tingling at the needling site and might radiate along the corresponding meridian). Then, an auxiliary needle $(13 \mathrm{~mm}$ in length and $0.18 \mathrm{~mm}$ in diameter) will be inserted, $2 \mathrm{~mm}$ lateral to the first needle, to a depth of $2 \mathrm{~mm}$ without achieving Deqi. Electrical stimulation will be applied at every acupoint, with one electrode connected to the filiform needle and the other to the auxiliary needle. This method circumscribes the electrical stimulation nearby, rather than going across the human body surface to cause performance bias. Electrical stimulation will last for $30 \mathrm{~min}$ in each acupuncture session. Electroacupuncture treatment will be performed three times per week from the fifth day of menstruation or withdrawal bleeding until before the next menstruation for three menstrual cycles. 


\section{Outcome measurements}

\section{Primary outcome}

The primary outcome will be the ovulation rate.

\section{Secondary outcomes}

The secondary outcomes will include the dominant follicle rate, mean number of dominant follicles, endometrial thickness, time point of ovulation, follicular size before ovulation, luteinizing hormone (LH) level, estradiol (E2) level, and pregnancy rate.

\section{Time points of outcome measurement}

Outcomes will be assessed at two time points: baseline and the completion of the treatment. Furthermore, patients will be given some regular tests at baseline, including routine blood tests, liver function test, and kidney function test. The overview of the outcome measurement at the different time points is shown in Table 1.

\section{Assessment of adverse events}

According to previous RCTs, acupuncture may cause several types of adverse events, including subcutaneous hematoma, bleeding, skin bruising, and needle site pain.

Table 1 Trial process chart

\begin{tabular}{|c|c|c|}
\hline Time point & Baseline & Post-treatment \\
\hline \multicolumn{3}{|l|}{ Diagnosis } \\
\hline Inclusion confirmed & $\checkmark$ & \\
\hline Informed consent signed & $\checkmark$ & \\
\hline Body sign & $\checkmark$ & $\checkmark$ \\
\hline Disease history & $\checkmark$ & \\
\hline Treatment history & $\checkmark$ & \\
\hline Comorbidity & $\checkmark$ & $\checkmark$ \\
\hline Current treatment & $\checkmark$ & $\checkmark$ \\
\hline \multicolumn{3}{|l|}{ Outcome assessment } \\
\hline B ultrasound & $\checkmark$ & $\checkmark$ \\
\hline Follicle-stimulating hormone & $\checkmark$ & $\checkmark$ \\
\hline Luteinizing hormone & $\checkmark$ & $\checkmark$ \\
\hline Testosterone & $\checkmark$ & $\checkmark$ \\
\hline Estradiol & $\checkmark$ & $\checkmark$ \\
\hline Blood HCG & $\checkmark$ & $\checkmark$ \\
\hline \multicolumn{3}{|l|}{ Regular tests } \\
\hline Routine blood tests & $\checkmark$ & \\
\hline Liver function test & $\checkmark$ & \\
\hline Kidney function test & $\checkmark$ & \\
\hline \multicolumn{3}{|c|}{ Data collection and statistical analysis } \\
\hline Adverse event & & $\checkmark$ \\
\hline Causes of dropout & & $\checkmark$ \\
\hline Safety analysis & & $\checkmark$ \\
\hline Compliance analysis & & $\checkmark$ \\
\hline
\end{tabular}

Among these adverse events, subcutaneous hematoma and bleeding were the most common [20]. Acupuncturerelated adverse events, including bleeding, hematoma, fainting, serious pain, local infection, etc., will be carefully recorded in the case report forms.

\section{Sample size calculation}

A previous study indicated that $59 \%$ of women with PCOS ovulated after receiving clomiphene citrate [11]. In this trial, the ovulation rate is expected to be $75 \%$ in the EA group and $55 \%$ in the CC group. Considering a significant level of 0.05 and a power of $0.80,50$ participants in each group are required, as calculated by $t$ test in G*Power (Version 3, Institute for Experimental Psychology, Heinrich-Heine-University, Germany). At least 58 participants per group and 116 total participants will be recruited to allow for a $15 \%$ dropout rate.

\section{Statistical analysis}

A statistician blinded to the whole trial process will perform the statistical analyses using the SAS statistical software package (Version 9.1, SAS Institute Inc.) in the Computer Integrated Manufacturing System. For the evaluation of a curative effect in this trial, the full analysis set (FAS) will be used. The FAS is determined according to an intention-to-treat (ITT) population; all randomized patients who receive at least one treatment session will be included in the analysis set. The perprotocol analysis set (PPS) is defined as the patients who complete the study and do not have major protocol violations. Demographic data and other basic indicators will be analyzed to test the balance of the two groups at baseline. The main indicators and global indicators will be analyzed within the FAS and PPS. The results will be described with mean, standard deviation, median, P25 (percentile 25), P75 (percentile 75), maximum, and minimum values of the differences between the treatment period and the baseline period. Between-group differences will be tested using repeated measure analyses of the variance. The accepted level of significance for all analyses was $P<0.05$. Considering the influences of other factors on the effectiveness, such as age, duration of disease, etc., these factors will be considered as covariants for the covariance analysis or logistic regression analysis when comparisons are made between the groups. Missing data will be replaced according to the principle of multiple imputations.

\section{Quality monitoring}

To control the trial's quality, an independent qualitymonitoring group will be established. The project leader will be in charge of the whole quality monitoring, whereas supervisors will be in charge of the quality monitoring in each center. The whole process will be 
supervised. All data will be verified to ensure they are recorded and reported accurately, authentically, completely, and consistently with the original data.

\section{Discussion}

The present trial is a comparative study of the effectiveness of electroacupuncture on ovulation and some related sex hormones in patients with PCOS.

Due to the weakness of the methodological design, the overall quality of clinical trials concerning the effectiveness of acupuncture treatment for infertility in women with PCOS is relatively low [18]. Although an ongoing multicenter RCT is underway, more RCTs that are properly designed are needed before drawing conclusions concerning the use of acupuncture in the management of PCOS [21]. Therefore, this trial is designed as an RCT to evaluate the add-on effects of electroacupuncture to conventional drugs on PCOS in terms of promoting ovulation and regulating related hormones. This study has several strengths.

On one hand, this trial is designed as an RCT. Due to its ability to reduce spurious causality and bias, the RCT is the most reliable scientific evidence in the hierarchy of evidence and can influence healthcare policy and practice. The RCT, the gold standard in clinical trials, is often used to test the efficacy or effectiveness of various types of medical interventions and may provide information about adverse effects. Therefore, this rigorously designed RCT, which will evaluate the effectiveness of electroacupuncture on PCOS in terms of promoting ovulation and regulating related hormones, will be able to provide some reliable evidence regarding the use of acupuncture for PCOS.

On the other hand, the therapeutic principle of treatment based on the phase of the menstrual cycle will be followed in this trial. In traditional Chinese Medicine, treatment based on syndrome differentiation is an important therapeutic principle. In gynecological diseases, treatment based on the phase of the menstrual cycle is another important principle and can enhance therapeutic effects. Therefore, this principle will be followed in our study. Sanyinjiao (SP 6), Zigong (EX-CA 1), Guanyuan (BL 26), and Zhongji (CV 3) have been chosen as the compulsory acupoints. These four acupoints are the ones most frequently used to treat PCOS, according to a text-mining analysis [22]. Sanyinjiao (SP 6) and Zigong (EX-CA 1) are important acupoints for the treatment of gynecological diseases in acupuncture practice. Guanyuan (BL 26) can reinforce kidney and warm Yang. Zhongji (CV 3) is located in the Conception Vessel and is used to regulate the Chong Vessel and Conception Vessel. In addition, according to a previous study [23], three sets of arbitrary points will be alternately selected according to the phase of the menstrual cycle, with each group consisting of two acupoints. After menstruation, Taichong (LR 3) and Taixi (KI 3) will be punctured to regulate qi and nourish the liver and kidneys. During the ovulatory period, Mingmen (GV 4) and Xuehai (SP 10) will be punctured to tonify qi and regulate the blood to promote ovulation. Before the next menstruation, Xuehai (SP 10) and Geshu (BL 17) will be punctured to regulate the blood.

This rigorously designed trial will allow for the collection of valuable and reliable data to evaluate the effectiveness of a specific acupuncture protocol for treating PCOS. This study will contribute to the clinical evidence regarding the effectiveness of electroacupuncture for women with PCOS.

\section{Trial status}

The trial had not yet begun at the time of manuscript submission.

\section{Abbreviations \\ PCOS: polycystic ovary syndrome; EA: electroacupuncture; CC: clomiphene citrate; LH: luteinizing hormone; E2: estradiol; FSH: follicle-stimulating hormone; RCT: randomized controlled trial; CONSORT: Consolidated Standards of Reporting Trials; STRICTA: Standards for Reporting Interventions in Controlled Trials of Acupuncture; ESHRE: European Society of Human Reproduction and Embryology; ASRM: American Society for Reproductive Medicine; SAS: Statistical Analysis System; FAS: full analysis set; ITT: intention- to-treat; PPS: per-protocol analysis set; P25: percentile 25; P75: percentile 75.}

\section{Competing interests}

The authors declare that they have no competing interests.

\section{Authors' contributions}

JC and SWF contributed equally to the manuscript. JC conceived the study, participated in its design, and drafted the manuscript. SWF participated in the design and drafted the manuscript. JZZ, XW, MXY, HZT, and HYF participated in the design and helped to draft the manuscript. JY and FRL conceived the study, participated in its design, and helped to draft the manuscript. All authors read and approved the final manuscript.

\section{Acknowledgements}

This trial was financially supported by funds from the Science and Technology Department of Sichuan Province (grant number 2015SZ0096).

\section{Author details}

${ }^{1}$ Chengdu University of Traditional Chinese Medicine, Chengdu, Sichuan, China. 'Maternal and Child Health Hospital of Sichuan Province, Chengdu, Sichuan, China. ${ }^{3}$ Sichuan Orthopedic Hospital, Chengdu, Sichuan, China.

Received: 10 December 2015 Accepted: 15 April 2016

Published online: 21 May 2016

\section{References}

1. Escobar-Morreale HF, Carmina E, Dewailly D, Gambineri A, Kelestimur F, Moghetti $P$, et al. Epidemiology, diagnosis and management of hirsutism: a consensus statement by the Androgen Excess and Polycystic Ovary Syndrome Society. Hum Reprod Update. 2012;18(2):146-70.

2. Joham AE, Teede HJ, Ranasinha S, Zoungas S, Boyle J. Prevalence of infertility and use of fertility treatment in women with polycystic ovary syndrome: data from a large community-based cohort study. J Womens Health. 2015;24(4):299-307.

3. Boomsma CM, Eijkemans MJ, Hughes EG, Visser GH, Fauser BC, Macklon NS. A meta-analysis of pregnancy outcomes in women with polycystic ovary syndrome. Hum Reprod Update. 2006;12(6):673-83.

4. Moran $\mathrm{L}$, Teede $\mathrm{H}$. Metabolic features of the reproductive phenotypes of polycystic ovary syndrome. Hum Reprod Update. 2009;15(4):477-88. 
5. Marcondes JA, Hayashida SA, Barcellos CR, Rocha MP, Maciel GA, Baracat EC. Metabolic syndrome in women with polycystic ovary syndrome: prevalence, characteristics and predictors. Arq Bras Endocrinol Metabol. 2007:51(6):972-9.

6. Legro RS, Kunselman AR, Dodson WC, Dunaif A. Prevalence and predictors of risk for type 2 diabetes mellitus and impaired glucose tolerance in polycystic ovary syndrome: a prospective, controlled study in 254 affected women. J Clin Endocrinol Metab. 1999;84(1):165-9.

7. Deeks AA, Gibson-Helm ME, Teede HJ. Anxiety and depression in polycystic ovary syndrome: a comprehensive investigation. Fertil Steril. 2010;93(7): 2421-3.

8. Himelein MJ, Thatcher SS. Polycystic ovary syndrome and mental health: A review. Obstet Gynecol Surv. 2006;61(11):723-32.

9. Vos T, Flaxman AD, Naghavi M, Lozano R, Michaud C, Ezzati M, et al. Years lived with disability (YLDs) for 1160 sequelae of 289 diseases and injuries 1990-2010: a systematic analysis for the Global Burden of Disease Study 2010. Lancet. 2012;380(9859):2163-96.

10. March WA, Moore VM, Willson KJ, Phillips DI, Norman RJ, Davies MJ. The prevalence of polycystic ovary syndrome in a community sample assessed under contrasting diagnostic criteria. Hum Reprod. 2010;25(2):544-51.

11. Zain MM, Jamaluddin R, Ibrahim A, Norman RJ. Comparison of clomiphene citrate, metformin, or the combination of both for first-line ovulation induction, achievement of pregnancy, and live birth in Asian women with polycystic ovary syndrome: a randomized controlled trial. Fertil Steril. 2009; 91(2):514-21.

12. Legro RS, Barnhart HX, Schlaff WD, Carr BR, Diamond MP, Carson SA, et al. Clomiphene, metformin, or both for infertility in the polycystic ovary syndrome. N Engl J Med. 2007;356(6):551-66.

13. Johansson J, Redman L, Veldhuis PP, Sazonova A, Labrie F, Holm G, et al. Acupuncture for ovulation induction in polycystic ovary syndrome: a randomized controlled trial. Am J Physiol Endocrinol Metab. 2013;304(9): E934-43.

14. Zheng YH, Wang XH, Lai MH, Yao H, Liu H, Ma HX. Effectiveness of abdominal acupuncture for patients with obesity-type polycystic ovary syndrome: a randomized controlled trial. J Altern Complement Med. 2013; 19(9):740-5.

15. Stener-Victorin E, Holm G, Janson PO, Gustafson D, Waern M. Acupuncture and physical exercise for affective symptoms and health-related quality of life in polycystic ovary syndrome: secondary analysis from a randomized controlled trial. BMC Complement Altern Med. 2013;13:131.

16. Maliqueo M, Benrick A, Alvi A, Johansson J, Sun M, Labrie F, et al. Circulating gonadotropins and ovarian adiponectin system are modulated by acupuncture independently of sex steroid or beta-adrenergic action in a female hyperandrogenic rat model of polycystic ovary syndrome. Mol Cell Endocrinol. 2015;412:159-69.

17. Jedel E, Labrie F, Oden A, Holm G, Nilsson L, Janson PO, et al. Impact of electro-acupuncture and physical exercise on hyperandrogenism and oligo/amenorrhea in women with polycystic ovary syndrome: a randomized controlled trial. Am J Physiol Endocrinol Metab. 2011;300(1): E37-45.

18. Lim DC, Chen W, Cheng LN, Xue CC, Wong FW, O'Sullivan AJ, et al. Acupuncture for polycystic ovarian syndrome. Cochrane Database Syst Rev. 2011:8:CD007689.

19. Rotterdam EA-SPCWG. Revised 2003 consensus on diagnostic criteria and long-term health risks related to polycystic ovary syndrome. Fertil Steril. 2004;81(1):19-25.

20. Zhao L, Zhang FW, Li Y, Wu X, Zheng H, Cheng LH, et al. Adverse events associated with acupuncture: three multicentre randomized controlled trials of 1968 cases in China. Trials. 2011;12:87.

21. Kuang H, Li Y, Wu X, Hou L, Wu T, Liu J, et al. Acupuncture and clomiphene citrate for live birth in polycystic ovary syndrome: study design of a randomized controlled trial. eCAM. 2013;2013:527303.

22. Pu J. Acupoints analysis by data mining in polycystic ovary syndrome and its related symptom. Heilongjiang: Heilongjiang University Of Chinese Medicine; 2012.

23. Wu K. Random controlled prospective study of the treatment for PCOS by acupuncture and Chinese medicine combined. Guangdong: Guangzhou University of Chinese Medicine; 2011.

\section{Submit your next manuscript to BioMed Central and we will help you at every step:}

- We accept pre-submission inquiries

- Our selector tool helps you to find the most relevant journal

- We provide round the clock customer support

- Convenient online submission

- Thorough peer review

- Inclusion in PubMed and all major indexing services

- Maximum visibility for your research

Submit your manuscript at www.biomedcentral.com/submit
C Biomed Central 